\title{
Mandibular second premolar with four roots
}

Sefika Nur Akyuz, Ali Erdemir

Department of Endodontics, Faculty of Dentistry, Kirikkale University, Turkey
Address for correspondence:

Dr. Sefika Nur Akyuz,

Department of Endodontics, Faculty of Dentistry, Kirikkale University, Turkey.

E-mail:nurakyuz@yahoo.com

\begin{abstract}
Detection of normal and abnormal variation in tooth anatomy is essential for clinical success. It is generally well known that the mandibular second premolar teeth have a single root and canal. However, the mandibular second premolar teeth have sometimes more than one root and root canal. The endodontic treatment of a mandibular second premolar with four roots which separated at different levels along the middle third of the root is presented in this case report. Preoperative radiographs appeared radiolucency and different root anatomy in the region of the mandibular second premolar. The root canals were prepared using Mtwo rotary system (VDW, Munich, Germany) and obturated laterally condensed gutta percha and AH plus (Dentsply De Trey, Konstanz, Germany). After the completion of root canal treatment, the tooth was restored with a posterior composite filling material. On follow-up, the tooth was clinically and radiographically asymptomatic for two years. Clinicians should be aware of the importance of careful clinical and radiographic examination of mandibular premolars during the endodontic treatment. Radiographs exposed at two different horizontal angles and their careful interpretation facilitates the search of additional root canals.
\end{abstract}

Key words

Mandibular premolars, multiple roots and root canals, periapical radiographs, root canal therapy, root canal morphology

\section{INTRODUCTION}

To obtain for a successful treatment outcome, biomechanical instrumentation and obturation of the root canal system are required. Lack of knowledge about the anatomy of root canal space and presence of untreated canal may be a reason for failure of root canal therapy. ${ }^{[1]}$

Variation in root canal morphology was suggested as the most likely reason for the high frequency of endodontic flare-ups and failures. ${ }^{[1,2]}$ It was reported that the most significant cause for endodontic failures was incomplete canal instrumentation, followed by incorrect canal obturation ${ }^{[2]}$ was indicated that the mandibular premolars was the most difficult teeth to treat endodontically because of the variations in canal anatomy. The presence of multiple roots and canals in mandibular premolars has been shown in different studies. ${ }^{[3-5]}$ Studies have reported that the incidence of two or more canals in the second mandibular premolars

\begin{tabular}{|l|l|}
\hline \multicolumn{2}{|c|}{ Access this article online } \\
\hline Quick Response Code: & Website: \\
\hline & www.ejgd.org \\
\cline { 2 - 3 } & \\
\hline
\end{tabular}

can vary between 1.2 and 34\%. ${ }^{[3]}$ Zillich and Dowson ${ }^{[3]}$ reported that three canals occured in $0.4 \%$ of the teeth. Chan et al..$^{[4]}$ reported a case with three canals, whilst Serman and Hasselgren ${ }^{[5]}$ showed radiographic evidence of multiple roots in mandibular premolars.

The following case report describes the endodontic treatment of a mandibular second premolar with four canals and emphasized the importance of careful clinical and radiographic examination during endodontic treatment.

\section{CASE REPORT}

A 44-year-old female patient with a noncontributory medical history was referred to the Department of Endodontics, Faculty of Dentistry, Kirikkale University for the treatment of painful left mandibular second premolar tooth. Clinical evaluation revealed extensive seconder caries under old bridge restoration associated with the mandibular second premolar. The tooth was slight sensitive to percussion and palpation. The hyperemia, swelling or sinus tract was not observed in the apical region of the tooth. The mobility was in normal limit and the probing with a periodontal probe did not reveal any periodontal pocket. The old bridge restoration was removed and the pulp vitality was measured. The tooth did not respond to thermal and electric pulp testing. A preoperative radiographic examination revealed that 
the carious lesion was in close proximity to the pulp chamber. There was a well-defined periapical lesion in the apical region and an irregular root morphology consisting of at multiple distinct roots in the middle region [Figure 1]. The diagnosis was made as chronic apical periodontitis with necrotic pulp for the tooth.

The patient was given a local anesthetic by periapical infiltration ( $2 \%$ lidocaine with 1:100000 epinephrine). The tooth was isolated with a rubber dam. The caries was removed and conventional endodontic access cavity was prepared. The pulp tissue was removed using barbed broaches and the pulp chamber was irrigated with $5.25 \%$ sodium hypochlorite. The coronal portion of the root was enlarged using gates-glidden drills. The patency of four root canals in the middle third of the root was detected by using the size of $10 \mathrm{k}$-files. The working length of the root canals was determined using an electronic apex locator (Root ZX, J. Morita Corp., Kyoto, Japan) and it was verified with radiographs. They were confirmed the presence of four roots, mesiobuccal, mesiolingual, distobuccal, and distolingual [Figure 2]. The root canals were sequentially prepared using nickel-titanium rotary instruments (Mtwo, VDW, Munich, Germany). During the root canal preparation, Mtwo 20/.06 instrument was broken in apical third of distobuccal root. A titanium ProUltra tip \#8 (Dentsply, Maillefer, Ballaigues, Switzerland) was used to loosen the instrument. In the meantime, copious irrigation with $5 \%$ sodium hypochlorite was performed but broken instrument could not be removed or by-passed. Copious amounts of $5.25 \%$ sodium hypochlorite solution along with RC-Prep (Premier Dental Philadelphia, PA, USA) were used for root canal irrigation and lubrication. After the instrumentation, the root canals were irrigated with $5.25 \%$ sodium hypochlorite and followed by $17 \%$ EDTA. They were dried with sterile paper points and packed with chlorhexidine calcium hydroxide combination. A sterile cotton pellet was placed in the pulp chamber and a temporary filling material (Cavit, ESPE, Seefeld, Germany) was used to close the access cavity.

After 2 weeks, the symptoms had subsided and the patient had no complaint of pain. The dressing and calcium hydroxide were removed and the canals were thoroughly cleaned by alternate irrigations of $17 \%$ EDTA and $5.25 \%$ sodium hypochlorite. The gutta perchas with a tug back sensation were selected for root canals and a control radiograph was taken with mastercones length. The root canals were obturated using AH Plus root canal sealer (Dentsply, De Trey, Konstanz, Germany) and laterally condensed gutta percha. Radiographs were taken to confirm the quality of the obturation [Figure 3]. The restoration of the tooth was completed using a self etching dentin bonding system (SE Bond, Kuraray, Osaka, Japan) and a composite resin (Clearfill Photoposterior, Kuraray, Osaka, Japan). The patient was orientated to the prosthetic restoration clinic for new bridge restoration and recalled for clinical and radiographic control.

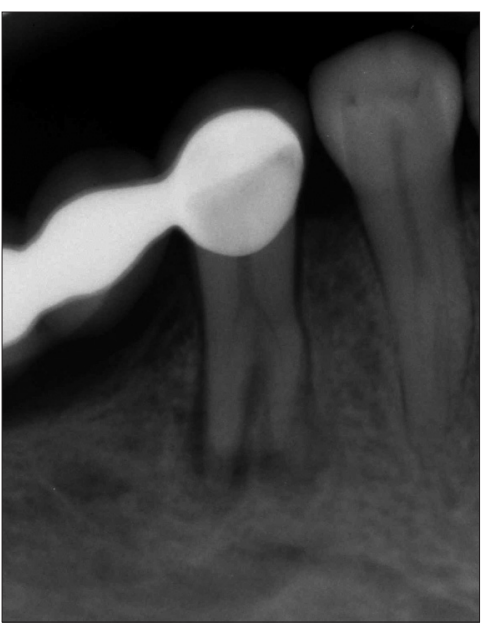

Figure 1: Preoperative radiograph of the mandibular second premolar

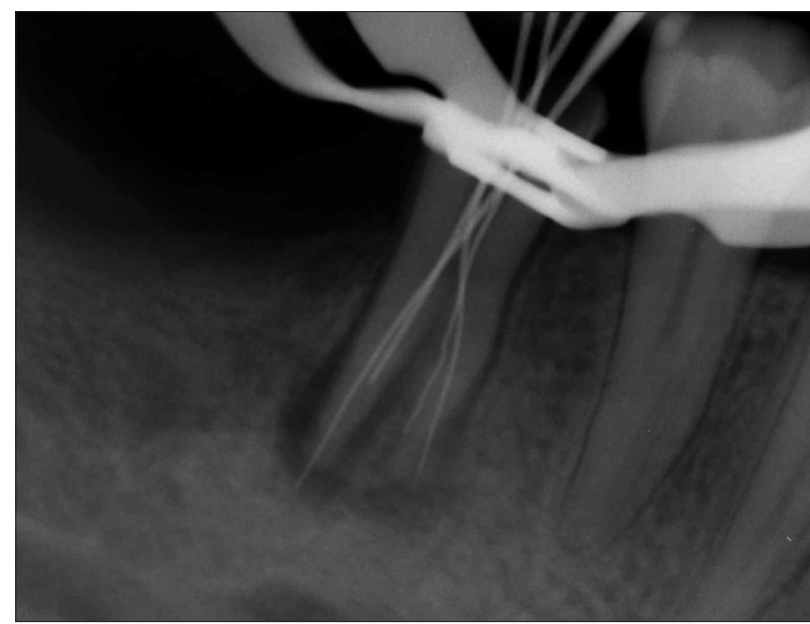

Figure 2: Radiography showing the presence of four roots, mesiobuccal, mesiolingual, distobuccal, and distolingual

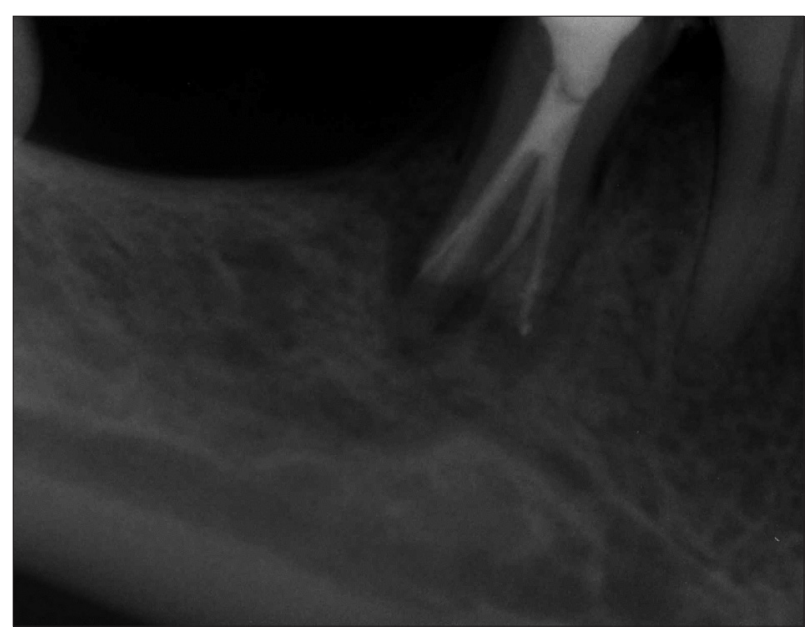

Figure 3: Mesially angulated radiograph showing completed root canal obturation

When the patient was referred to endodontic clinic after 2 year, the tooth was asymptomatic clinically and the control radiographs were revealed that the radioluceny 
in the apical region of the mandibular second premolar had disappeared [Figure 4].

In this case, when the patient's bilateral teeth evaluated radiographically, sudden disappearance were seen in the right first premolar tooth but not for the right second premolar [Figure 5].

\section{DISCUSSION}

For a successful treatment outcome in endodontics, it is essential to reach, clean and shape the root canals properly before a hermetic filling. ${ }^{[2]}$ The presence of an untreated additional canals leads to failure of endodontic treatment, it is an important factor that may be encountered with mandibular premolars. ${ }^{[6]}$

A large number of case reports have been published which describe a variety of complex canal configurations of mandibular premolars..$^{[3,7,8]}$ Various studies mentioned that disability to detect, locate, negotiate and instrument all root canals may lead to endodontic failure. ${ }^{[4]}$ Knowledge of basic root canal morphology of the mandibular second premolar as well as its variations was essential in the treatment of this tooth. ${ }^{[6]}$ Mandibular second premolar is one of the most difficult teeth for the endodontic treatment. ${ }^{[9]}$ This can be attributed to variations in the internal morphology of their pulp cavity, considering the number of root canals, apical deltas and lateral canals. ${ }^{[10]}$ It is probable that if all root canals had not been instrumented and obturated, a successful result may not have been achieved. Several clinical and experimental studies have documented failure to recognize a third and fourth canal might have resulted in incomplete treatment and leads to endodontic failure. ${ }^{[1]}$

Current methods such as canal staining and clearing techniques, ${ }^{[12]}$ transverse crosssectioning., ${ }^{[13,14]}$ radiographical examination ${ }^{[15]}$ dentine troughing under magnification, ${ }^{[12]}$ clinical operating microscope, ${ }^{[16]}$

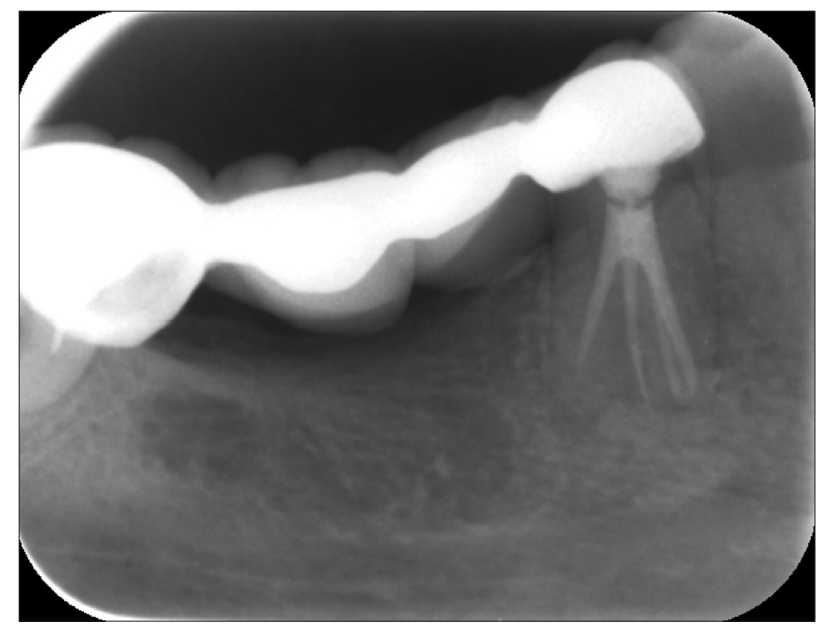

Figure 4: 2 year follow up control radiography showing completed healing ultrasonics, ${ }^{[17]}$ scanning electron microscope, ${ }^{[18]}$ cone-beam computed tomography $(\mathrm{CBCT})^{[19]}$ and microcomputed tomography are used in laboratory studies to demonstrate variations in canal anatomy. ${ }^{20]}$ But, use of operating microscope ${ }^{[21]}$ and conventional radiography ${ }^{[22]}$ are most widely methods at various stages of endodotic treatment to identify the complexity in the root canal morphology.

Conventional periapical radiographs are essential for preoperative diagnosis. Careful interpretation of two or more angled periapical radiographs provide much needed information about complex root canal morphology. ${ }^{[7]}$ In mandibular second premolars only the 40 degree horizontal angle identified the correct morphology. ${ }^{[2]}$ It was emphasized that critical importance of carefully evaluating each radiograph taken prior to treatment. ${ }^{[7]}$ In our case, diagnostic periapical radiography revealed multiple roots at the middle third of the root and orifices were close to each other. Thus, during the treatment negotiation and instrumentation of the root canals was difficult due to the root canal furcation at the mid-root level.

Mandibular premolars show a wide variety of root canal anatomy which must be considered at the beginning of treatment. Careful radiographical evaluation of the root and periodontal ligament outlines can help determine unusual anatomy. Sudden disappearance or narrowing of the root canal space can indicate that it divides. ${ }^{[6]}$ Evaluating the radiographs of left mandibular second premolar, in this case can cause one to suspect an unusual anatomy. Sabala et al. ${ }^{[23]}$ discovered that aberrations occurred under $1 \%$ of the cases and $90 \%$ of such aberrations were bilateral. In this case, when the patient's bilateral teeth evaluated radiographically, sudden disappearance were seen in the right first premolar tooth but not for the right second premolar.

In the presented case report, the 2 -year recall radiographs showed healing of the periradicular lesions, there were

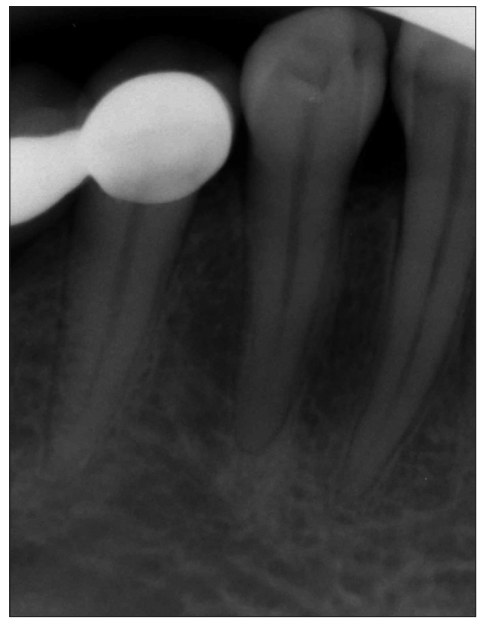

Figure 5: Bilateral teeth radiography 
no clinical symptoms or radiological changes such as periapical lesion. It is probable that if all the canals had not been properly instrumented, cleaned and obturated, then such success would not have been achieved.

The purpose of endodontic treatment is thorough debridement of the root canals followed by its obturation. In order to treat teeth with complex root canal morphology, a thorough and methodical approach is needed. The use of multi-angled diagnostic radiographs and operating microscope may be very useful in the diagnosis and necessary to locate and successfully treat unusual canal anatomies.

\section{REFERENCES}

1. Cleghorn BM, Christie WH, Dong CC. The root and root canal morphology of the human mandibular first premolar: A literature review. J Endod 2007;33:509-16.

2. Slowey RR. Root canal anatomy. Road map to successful endodontics. Dent Clin North Am 1979;23:555-73.

3. Zillich R, Dowson J. Root canal morphology of mandibular first and second premolars. Oral Surg Oral Med Oral Pathol 1973;36:738-44.

4. Chan K, Yew SC, Chao SY. Mandibular premolar with three root canals--two case reports. Int Endod J 1992;25:261-4.

5. Serman NJ, Hasselgren G. The radiographic incidence of multiple roots and canals in human mandibular premolars. Int Endod $\mathrm{J}$ 1992;25:234-7.

6. Vertucci FJ, Gegauff A. Root canal morphology of the maxillary first premolar. J Am Dent Assoc 1979;99:194-8.

7. Rodig T, Hulsmann M. Diagnosis and root canal treatment of a mandibular second premolar with three root canals. Int Endod $\mathrm{J}$ 2003;36:912-9.

8. Li X, Liu N, Liu R, Dong Z, Liu L, Deng M. Comparative study of root canal morphology of mandibular first premolar by micro-CT and radio visio graphy. Hua Xi Kou Qiang Yi Xue Za Zhi 2012;30:57-60.

9. Vertucci FJ, Williams RG. Root canal anatomy of the mandibular first molar. J Dent Assoc 1974;45:27-8.

10. Vertucci FA, Francois KJ. Endodontic therapy of a mandibular second premolar: a case report with clinical correlations. Fla Dent J 1986;57:25-7.

11. Sherwood IA. Pre-operative diagnostic radiograph interpretation by general dental practitioners for root canal treatment. Dentomaxillofac Radiol 2012;41:43-54.

12. Yoshioka T, Kikuchi I, Fukumoto Y, Kobayashi C, Suda H. Detection of the second mesiobuccal canal in mesiobuccal roots of maxillary molar teeth ex vivo. Int Endod J 2005;38:124-8.

13. Schwarze T, Baethge C, Stecher T, Geurtsen W. Identification of second canals in the mesiobuccal root of maxillary first and second molars using magnifying loupes or an operating microscope. Aust Endod J 2002;28:57-60.

14. Kulild JC, Peters DD. Incidence and configuration of canal systems in the mesiobuccal root of maxillary first and second molars. J Endod 1990;16:311-7.

15. Fava LR, Dummer PM. Periapical radiographic techniques during endodontic diagnosis and treatment. Int Endod J 1997;30:250-61.

16. Baldassari-Cruz LA, Lilly JP, Rivera EM. The influence of dental operating microscope in locating the mesiolingual canal orifice. Oral Surg Oral Med Oral Pathol Oral Radiol Endod 2002;93:190-4.

17. Alacam T, Tinaz AC, Genc O, Kayaoglu G. Second mesiobuccal canal detection in maxillary first molars using microscopy and ultrasonics. Aust Endod J 2008;34:106-9.

18. Gilles J, Reader A. An SEM investigation of the mesiolingual canal in human maxillary first and second molars. Oral Surg Oral Med Oral Pathol 1990;70:638-43.

19. Matherne RP, Angelopoulos C, Kulild JC, Tira D. Use of cone-beam computed tomography to identify root canal systems in vitro. J Endod 2008;34:87-9.

20. Plotino G, Grande NM, Pecci R, Bedini R, Pameijer CH, Somma F. Three-dimensional imaging using microcomputed tomography for studying tooth macromorphology. J Am Dent Assoc 2006;137: 1555-61.

21. Sempira HN, Hartwell GR. Frequency of second mesiobuccal canals in maxillary molars as determined by use of an operating microscope: A clinical study. J Endod 2000;26:673-4.

22. Omer OE, Al Shalabi RM, Jennings M, Glennon J, Claffey NM. A comparison between clearing and radiographic techniques in the study of the root-canal anatomy of maxillary first and second molars. Int Endod J 2004;37:291-6.

23. Sabala CL, Benenati FW, Neas BR. Bilateral root or root canal aberrations in a dental school patient population. J Endod 1994;20:38-42.

How to cite this article: Akyuz SN, Erdemir A. Mandibular second premolar with four roots. Eur J Gen Dent 2012;1:54-7.

Source of Support: Nil, Conflict of Interest: None declared. 